\title{
LIE THEORETIC ORIGIN OF SOME GENERATING FUNCTIONS OF FOX'S H-FUNCTION
}

\author{
RENU JAIN, D. K. JAIN AND P. K. CHATURVEDI
}

\begin{abstract}
The group theoretic method for achieving unification of diverse mass of literature of special functions is most recent of such efforts and is definitely the most elegant one. In this method the special functions emerge as basis vectors and matrix elements of local multiplier representation of some well known groups. This dual role played by special functions affords a powerful technique for derivation of several generating functions and addition theorems for them.

The present paper aims at harnessing this technique to generate, derive and interpret certain expansion of Fox's H-function in series of $\mathrm{H}$-function. In the special case these expansions reduce to corresponding results for G-function.
\end{abstract}

\section{Introduction}

Lie theoretic techniques are being employed since 1950's to achieving unification in heterogeneous growth of literature on special functions.

One of the foremost applications of Lie theory to special functions is for derivation of their generating functions. On the basis of differential recurrence relations satisfied by a given special function a Lie algebra of differential operators is constructed. The local multiplier representation for the corresponding Lie group then yields generating relation for corresponding special functions.

Miller W. $[4,5]$ has done most noteworthy work in this direction and has obtained generating functions for hypergeometric functions, Lauricella functions and Meijer's G-functions, besides undertaking exhaustive theoretical investigations which have gone a long way in establishing the significance of group theoretic techniques. In the present paper a Lie algebra has been constructed on the basis of differential recurrence relations satisfied by $\mathrm{H}$-function. Then multiplier representation for one parameter subgroup is employed to derive a generating relation for those functions.

Corresponding author: Renu Jain.

2010 Mathematics Subject Classification. 33C60, 33C45.

Key words and phrases. Lie algebra, H-function, G-function and generating function. 


\section{The H-function}

The Fox's H-function is defined as [2]

$$
\begin{aligned}
H_{p, q}^{m, n}[z] & =H_{p, q}^{m, n}\left[z \mid \begin{array}{c}
\left(a_{p}, \alpha_{p}\right) \\
\left(b_{q}, \beta_{q}\right)
\end{array}\right]=H_{p, q}^{m, n}\left[z\left[\begin{array}{l}
\left\{\left(a_{j}, \alpha_{j}\right)_{1, p}\right\} \\
\left\{\left(b_{j}, \beta_{j}\right)_{1, q}\right\}
\end{array}\right]\right. \\
& =\frac{1}{2 \pi \omega} \int_{L} \frac{\prod_{j=1}^{m} \Gamma\left(b_{j}-\beta_{j} s\right) \prod_{j=1}^{n} \Gamma\left(1-a_{j}+\alpha_{j} s\right)}{\prod_{j=m+1}^{q} \Gamma\left(1-b_{j}+\beta_{j} s\right) \prod_{j=n+1}^{p} \Gamma\left(a_{j}-\alpha_{j} s\right)} Z^{s} d s
\end{aligned}
$$

Here $\omega$ is square root of -1 the detailed conditions of formation are referred to in the literature [2].

Following differential recurrence relations are obeyed by Fox's H-function $[6,8]$.

$$
\left(z \partial z-\frac{\left(a_{j}-1\right)}{\alpha_{j}}\right) H_{p, q}^{m, n}\left[z \mid \begin{array}{c}
\left(a_{p}, \alpha_{p}\right) \\
\left(b_{q}, \beta_{q}\right)
\end{array}\right]= \pm \frac{1}{\alpha_{j}} H_{p, q}^{m, n}\left[z \mid \begin{array}{c}
\left(a_{j}-1, \alpha_{j}\right) \\
\left(b_{q}, \beta_{q}\right)
\end{array}\right]
$$

(signs are positive or negative according to $1 \leq j \leq n$ or $n+1 \leq j \leq p$ ).

$$
\left(z \partial z-\frac{b_{k}}{\beta_{k}}\right) H_{p, q}^{m, n}\left[z \mid \begin{array}{c}
\left(a_{p}, \alpha_{p}\right) \\
\left(b_{q}, \beta_{q}\right)
\end{array}\right]=\mp \frac{1}{\beta_{k}} H_{p, q}^{m, n}\left[z \mid \begin{array}{c}
\left(a_{p}, \alpha_{p}\right) \\
\left(b_{k}+1, \beta_{q}\right)
\end{array}\right]
$$

(if $1 \leq k \leq m$ sign would be negative and if $m+1 \leq k \leq \mathrm{q}$ sign would be positive). Also

$$
z H_{p, q}^{m, n}\left[z \mid \begin{array}{c}
\left(a_{p}, \alpha_{p}\right) \\
\left(b_{q}, \beta_{q}\right)
\end{array}\right]=H_{p, q}^{m, n}\left[z \mid \begin{array}{c}
\left(a_{p}+\alpha_{p}, \alpha_{p}\right) \\
\left(b_{q}+\beta_{q}, \beta_{q}\right)
\end{array}\right] .
$$

Introducing new parameters $t_{1}, t_{2}, \ldots, t_{p} ; u_{1}, u_{2}, \ldots, u_{q}$, we define the following basis functions.

$$
F_{p, q}^{m, n}\left[z \mid \begin{array}{c|c}
t_{P} & \left(a_{p}, \alpha_{p}\right) \\
u_{q} & \left(b_{q}, \beta_{q}\right)
\end{array}\right]=H_{p, q}^{m, n}\left[z \mid \begin{array}{c}
\left(a_{p}, \alpha_{p}\right) \\
\left(b_{q}, \beta_{q}\right)
\end{array}\right] t_{1}^{a_{1}} \cdots t_{p}^{a_{p}} \cdot u_{1}^{b_{1}} \cdots u_{q}^{b_{q}} .
$$

With the help of relations (2.1) and (2.2), we construct partial differential operators

$$
\begin{array}{rlrl}
T_{j} & =\frac{t_{j}}{\alpha_{j}} \partial t_{j} ; & L_{j}=t_{j}^{-1}\left(z \partial z-\frac{t_{j}}{\alpha_{j}} \partial t_{j}+\frac{1}{\alpha_{j}}\right) & \text { for } 1 \leq j \leq p ; \\
U_{k} & =\frac{u_{k}}{\beta_{k}} \partial u_{k} ; \quad R_{k}=u_{k}\left(z \partial z-\frac{u_{k}}{\beta_{k}} \partial u_{k}\right) & \text { for } 1 \leq k \leq q \text { and } \\
V & =z t_{1}^{a_{1}} \cdots t_{p}^{a_{p}} \cdot u_{1}^{b_{1}} \cdots u_{q}^{b_{q}} &
\end{array}
$$

These operators satisfy following commutation relations

$$
\left[T_{j}, L_{j}\right]=-\frac{L_{j}}{\alpha_{j}} ; \quad\left[U_{k}, R_{k}\right]=\frac{R_{K}}{\beta_{K}} ; \quad\left[T_{j}, V\right]=\left[U_{k}, V\right]=V .
$$


All other commutator relations of any two operators are zero. It can be easily verified that operators generate a $2(p+q)+1$ dimensional Lie-algebra. The actions of these operators on basis functions defined by equation (2.5) is given by

$$
\begin{aligned}
T_{j} F_{p, q}^{m, n}[z] & =\frac{a_{j}}{\alpha_{j}} F_{p, q}^{m, n}[z] \\
U_{k} F_{p, q}^{m, n}[z] & =\frac{b_{k}}{\beta_{k}} F_{p, q}^{m, n}[z] \\
L_{j} F_{p, q}^{m, n}[z] & = \pm \frac{1}{\alpha_{j}} F_{p, q}^{m, n}\left[z\left|\begin{array}{l}
t_{p} \\
u_{q}
\end{array}\right| \begin{array}{l}
\left.\left(a_{1}, \alpha_{1}\right), \ldots,\left(a_{j}-1, \alpha_{j}\right), \ldots,\left(a_{p}, \alpha_{p}\right)\right] \\
\left(b_{q}, \beta_{q}\right)
\end{array}\right]
\end{aligned}
$$

(plus sign corresponds to $1 \leq j \leq n$ and minus sign to $n+1 \leq j \leq p$ ).

$$
R_{k} F_{p, q}^{m, n}[z]=\mp \frac{1}{\beta_{k}} F_{p, q}^{m, n}\left[z\left|\begin{array}{l}
t_{p} \\
u_{q}
\end{array}\right| \begin{array}{l}
\left(a_{p}, \alpha_{p}\right) \\
\left(b_{1}, \beta_{1}\right), \ldots,\left(b_{k}+1, \beta_{k}\right), \ldots,\left(b_{q}, \beta_{q}\right)
\end{array}\right]
$$

(minus sign to be taken if $1 \leq k \leq m$ and plus sign to be taken if $m+1 \leq k \leq q$ ).

\section{Generating functions resulting from the multiplier representation for one parameter} subgroup exp $\left(\lambda L_{j}\right)$

By standard multiplier representation technique for obtaining generating relations for $\mathrm{H}$-functions we need computation of action of one parametric subgroup $\left(\lambda L_{j}\right)$. This can be done by usual techniques of multiplier representation theory [4]. Thus we obtain resulting transformations in the form

$$
\left[\begin{array}{c}
z \\
t_{j} \\
u_{k}
\end{array}\right] \rightarrow\left[\begin{array}{c}
z\left(1-\frac{\lambda}{\alpha_{j} t_{j}}\right)^{-\alpha_{j}} \\
t_{j}-\frac{\lambda}{\alpha_{j}} \\
u_{k}
\end{array}\right]
$$

Also the multiplier $v$ is given by

$$
v=\left(1-\frac{\lambda}{\alpha_{j} t_{j}}\right)^{-1}
$$

(i) Now we drive the multiplication theorems for Fox's H-functions corresponding to operator $L_{j}$, when $1 \leq j \leq n$.

By direct expansion, we get

$$
\begin{aligned}
& \exp \left(\lambda L_{j}\right) F_{p, q}^{m, n}\left[z\left|\begin{array}{c|c}
t_{p} \\
u_{q}
\end{array}\right| \begin{array}{l}
\left(a_{p}, \alpha_{p}\right) \\
\left(b_{q}, \beta_{q}\right)
\end{array}\right]=\sum_{r=0}^{\infty} \frac{\lambda^{r}}{r !}\left(L_{j}\right)^{r} F_{p, q}^{m, n}\left[z\left|\begin{array}{c}
t_{p} \\
u_{q}
\end{array}\right| \begin{array}{l}
\left(a_{p}, \alpha_{p}\right) \\
\left(b_{q}, \beta_{q}\right)
\end{array}\right] \\
& =\sum_{r=0}^{\infty} \frac{\lambda^{r}}{r !\left(\alpha_{j}\right)^{r}} F_{p, q}^{m, n}\left[z\left|\begin{array}{l}
t_{p} \\
u_{q}
\end{array}\right|_{\left(b_{q}, \beta_{q}\right)}^{\left(a_{1}, \alpha_{1}\right) \cdots\left(a_{j}-r, \alpha_{j}\right) \cdots\left(a_{p}, \alpha_{p}\right)}\right]
\end{aligned}
$$


$=\sum_{r=0}^{\infty} \frac{\lambda^{r}}{r !\left(\alpha_{j}\right)^{r}} H_{p, q}^{m, n}\left[\left.z\right|_{\left(b_{q}, \beta_{q}\right)} ^{\left(a_{1}, \alpha_{1}\right), \ldots,\left(a_{j}-r, \alpha_{j}\right), \ldots,\left(a_{p}, \alpha_{p}\right)}\right] t_{1}^{a_{1}} \cdots t_{j}^{a_{j}-r} \cdots t_{p}^{a_{p}} \cdot u_{1}^{b_{1}} \cdots u_{q}^{b_{q}}$.

On the other hand evaluating $\exp \left(\lambda L_{j}\right) F_{p, q}^{m, n}$ by the transformation formulae (3.1) and (3.2), we get

$$
\begin{aligned}
& \exp \left(\lambda L_{j}\right) F_{p, q}^{m, n}\left[z \mid \begin{array}{c}
t_{p} \\
u_{q}
\end{array} \begin{array}{c}
\left(a_{p}, \alpha_{p}\right) \\
\left(b_{q}, \beta_{q}\right)
\end{array}\right] \\
& =\left(1-\frac{\lambda}{\alpha_{j} t_{j}}\right)^{-1} H_{p, q}^{m, n}\left[\left.z\left(1-\frac{\lambda}{\alpha_{j} t_{j}}\right)^{-\alpha_{j}}\right|_{\left(\begin{array}{c}
\left(a_{p}, \alpha_{p}\right) \\
\left(b_{q}, \beta_{q}\right)
\end{array}\right] t_{1}^{a_{1}} \cdots\left(t_{j}-\frac{\lambda}{\alpha_{j}}\right)^{-a_{j}} \cdots t_{p}^{a_{p}} \cdot u_{1}^{b_{1}} \cdots u_{q}^{b_{q}} .}\right.
\end{aligned}
$$

Comparing these two values of $\exp \left(\lambda L_{j}\right) F_{p, q}^{m, n}[z]$, we arrive at the identity

$$
\begin{aligned}
& \left(1-\frac{\lambda}{\alpha_{j} t_{j}}\right)^{-1} H_{p, q}^{m, n}\left[\left.z\left(1-\frac{\lambda}{\alpha_{j} t_{j}}\right)^{-\alpha_{j}}\right|_{\left(\begin{array}{l}
\left(a_{p}, \alpha_{p}\right) \\
\left(b_{q}, \beta_{q}\right)
\end{array}\right]\left(t_{j}-\frac{\lambda}{\alpha_{j}}\right)^{-\alpha_{j}}}\right. \\
& \quad=\sum_{r=0}^{\infty} \frac{\lambda^{r}}{r !\left(\alpha_{j}\right)^{r}} H_{p, q}^{m, n}\left[z \mid \begin{array}{l}
\left(a_{1}, \alpha_{1}\right), \ldots,\left(a_{j}-r, \alpha_{j}\right), \ldots,\left(a_{p}, \alpha_{p}\right) \\
\left(b_{q}, \beta_{q}\right)
\end{array}\right] \times t_{j}^{a_{j}-r}
\end{aligned}
$$

Employing substitution $\left(1-\frac{\lambda}{\alpha_{j} t_{j}}\right)^{-\alpha_{j}}=\eta$, we get the generating relation

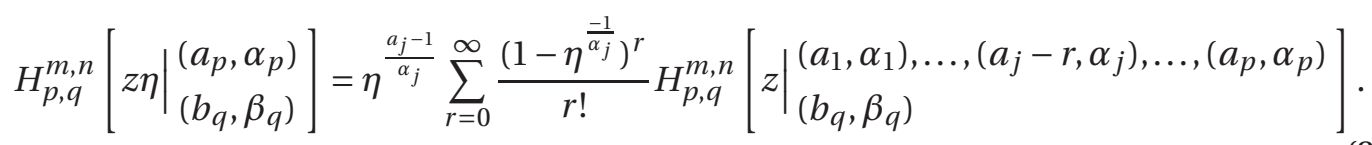

For $j=1$, this general result reduces to well known generating relation for Fox's H-function [7, (2.9.11); p. 25].

$$
\left.H_{p, q}^{m, n}[z \eta] \begin{array}{l}
\left(a_{p}, \alpha_{p}\right) \\
\left(b_{q}, \beta_{q}\right)
\end{array}\right]=\eta^{\frac{a_{1}-1}{\alpha_{1}}} \sum_{r=0}^{\infty} \frac{\left(1-\eta^{\frac{-1}{\alpha_{1}}}\right)^{r}}{r !} H_{p, q}^{m, n}\left[z \mid \begin{array}{l}
\left.\left(a_{1}-r, \alpha_{1}\right),\left(a_{j}, \alpha_{j}\right)_{2, p}\right] \\
\left(b_{k}, \beta_{k}\right)_{1, q}
\end{array}\right]
$$

Again particularizing (3.7), by substitution $\alpha_{j}=\beta_{k}=1$ and $\eta=\lambda$ it takes the form of a well known generating relation for Meijer's G-function [1, (3); p.213]

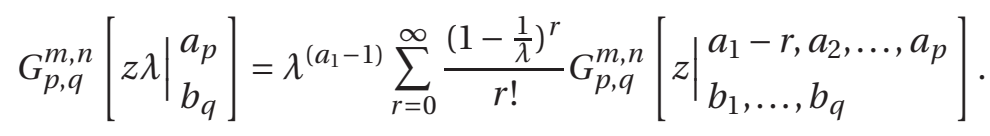

(ii) Similarly, considering the action of one parameter subgroup $\exp \left(\lambda L_{j}\right)$ generated by operator $L_{j}$ when $n+1 \leq j \leq p$, we arrive at the identity

$$
\left(1-\frac{\lambda}{\alpha_{j} t_{j}}\right)^{-1} H_{p, q}^{m, n}\left[\left.z\left(1-\frac{\lambda}{\alpha_{j} t_{j}}\right)^{-\alpha_{j}}\right|_{\left(b_{q}, \beta_{q}\right)} ^{\left(a_{p}, \alpha_{p}\right)}\right]\left(t_{j}-\frac{\lambda}{\alpha_{j}}\right)^{a_{j}}
$$




$$
=\sum_{r=0}^{\infty} \frac{(-\lambda)^{r}}{\left(\alpha_{j}\right)^{r} r !} H_{p, q}^{m, n}\left[z \mid \begin{array}{l}
\left(a_{1}, \alpha_{1}\right), \ldots,\left(a_{j}-r, \alpha_{j}\right), \ldots,\left(a_{p}, \alpha_{p}\right) \\
\left(b_{q}, \beta_{q}\right)
\end{array}\right] \times t_{j}^{a_{j}-r} .
$$

On substituting $\left(1-\frac{\lambda}{\alpha_{j} t_{j}}\right)^{-\alpha_{j}}=\eta$, equation (3.9) takes the form of the following generating function for Fox's H-function

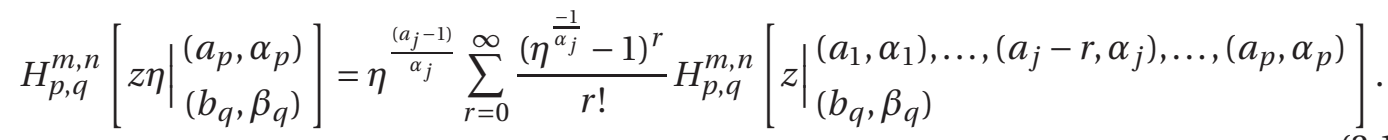

Now for $j=p$, the relati on (3.10) reduces to a well- known generating function [7, (2.9.9); p.25] for Fox's H-function

$$
H_{p, q}^{m, n}\left[z \eta \mid \begin{array}{c}
\left(a_{p}, \alpha_{p}\right) \\
\left(b_{q}, \beta_{q}\right)
\end{array}\right]=\eta^{\frac{a_{p}-1}{\alpha_{p}}} \sum_{r=0}^{\infty} \frac{\eta^{\frac{-1}{\alpha_{p}}}-1^{r}}{r !} H_{p, q}^{m, n}\left[\left.z\right|_{\left(b_{k}, \beta_{k}\right)_{1, q}} ^{\left(a_{j}, \alpha_{j}\right)_{1, p-1},\left(a_{p}-r, \alpha_{p}\right)}\right] .
$$

Further if we take $\alpha_{j}=\beta_{k}=1$ and $\eta=\lambda$ then result (20) reduces to well known generating function [1, (4); p. 213] for Meijer's G-function.

$$
G_{p, q}^{m, n}\left[\left.z \lambda\right|_{\begin{array}{l}
a_{p} \\
b_{q}
\end{array}}\right]=\lambda^{a_{p}-1} \sum_{r=0}^{\infty} \frac{1}{r !}\left(\frac{1}{\lambda}-1\right)^{r} G_{p, q}^{m, n}\left[z \mid \begin{array}{l}
a_{1}, a_{2}, \ldots, a_{p-1}, a_{p}-r \\
b_{1}, \ldots, b_{q}
\end{array}\right] .
$$

\section{Generating functions resulting from multiplier representation for one parameter sub-} group $\exp \left(\mu R_{k}\right)$

The action of one parameter subgroup $\exp \left(\mu R_{k}\right)$ on analytic function $R_{k}$ can be computed by employing usual techniques of multiplier representation theory [4].

The induced transformations are

$$
\left[\begin{array}{c}
z \\
t_{j} \\
u_{k}
\end{array}\right] \rightarrow\left[\begin{array}{c}
z\left(1+\frac{\mu u_{k}}{\beta_{k}}\right)^{\beta_{k}} \\
t_{j} \\
u_{k}\left(1+\frac{\mu u_{k}}{\beta_{k}}\right)^{-1}
\end{array}\right]
$$

Evaluating $\exp \left(\mu R_{k}\right) F_{p, q}^{m, n}[z]$ by direct expansion on one hand and using above transformation on the other, as was done in the previous section, we arrive at the identities.

$$
\begin{aligned}
& H_{p, q}^{m, n}\left[z\left(1+\frac{\mu u_{k}}{\beta_{k}}\right)^{\beta_{k}} \mid \begin{array}{l}
\left(a_{p}, \alpha_{p}\right) \\
\left(b_{q}, \beta_{q}\right)
\end{array}\right] u_{k}^{b_{k}}\left(1+\frac{\mu u_{k}}{\beta_{k}}\right)^{-b_{k}} \\
& \quad=\sum_{r=0}^{\infty} \frac{(-\mu)^{r}}{r !\left(\beta_{k}\right)^{r}} H_{p, q}^{m, n}\left[z \mid \begin{array}{l}
\left(a_{p}, \alpha_{p}\right) \\
\left(b_{1}, \beta_{1}\right), \ldots,\left(b_{k}+r, \beta_{k}\right), \ldots,\left(b_{m}, \beta_{m}\right),\left(b_{m+1}, \beta_{m+1}\right), \ldots,\left(b_{q}, \beta_{q}\right)
\end{array}\right] \times u_{k}^{b_{k}+r},
\end{aligned}
$$


when $1 \leq k \leq m$ and

$$
\begin{aligned}
& H_{p, q}^{m, n}\left[z\left(1+\frac{\mu u_{k}}{\beta_{k}}\right)^{\beta_{k}} \mid \begin{array}{c}
\left(a_{p}, \alpha_{p}\right) \\
\left(b_{q}, \beta_{q}\right)
\end{array}\right]\left(u_{k}\right)^{b_{k}}\left(1+\frac{\mu u_{k}}{\beta_{k}}\right)^{-b_{k}} \\
& \quad=\sum_{r=0}^{\infty} \frac{(\mu)^{r}}{r !\left(\beta_{k}\right)^{r}} H_{p, q}^{m, n}\left[z \mid \begin{array}{l}
\left(a_{p}, \alpha_{p}\right) \\
\left(b_{1}, \beta_{1}\right), \ldots,\left(b_{m}, \beta_{m}\right),\left(b_{m+1}, \beta_{m+1}\right),\left(b_{k}+r, \beta_{k}\right), \ldots,\left(b_{q}, \beta_{q}\right)
\end{array}\right] \times u_{k}^{b_{k}+r},
\end{aligned}
$$

when $m+1 \leq k \leq q$.

Using substitution $\eta=\left(1+\frac{\mu u_{k}}{\beta_{k}}\right)^{\beta_{k}}$ these identities take the form

$$
\begin{aligned}
& H_{p, q}^{m, n}\left[z \eta \mid \begin{array}{c}
\left(a_{p}, \alpha_{p}\right) \\
\left(b_{q}, \beta_{q}\right)
\end{array}\right] \\
& =\eta^{\frac{b_{k}}{\beta_{k}}} \sum_{r=0}^{\infty} \frac{\left(1-\eta^{\frac{1}{\beta_{k}}}\right)^{r}}{r !} H_{p, q}^{m, n}\left[\left.z\right|_{\left(b_{1}, \beta_{1}\right), \ldots,\left(b_{k}+r, \beta_{k}\right),\left(b_{m}, \beta_{m}\right),\left(b_{m+1}, \beta_{m+1}\right), \ldots,\left(b_{q}, \beta_{q}\right)}\right)
\end{aligned}
$$

when $1 \leq k \leq m$ and

$$
\begin{aligned}
& H_{p, q}^{m, n}\left[z \eta \mid \begin{array}{l}
\left(a_{p}, \alpha_{p}\right) \\
\left(b_{q}, \beta_{q}\right)
\end{array}\right] \\
& =\eta^{\frac{b_{k}}{\beta_{k}}} \sum_{r=0}^{\infty} \frac{\left(\eta^{\left.\frac{1}{\beta_{k}}-1\right)^{r}}\right.}{r !} H_{p, q}^{m, n}\left[z \mid \begin{array}{l}
\left(a_{p}, \alpha_{p}\right) \\
\left(b_{1}, \beta_{1}\right), \ldots,\left(b_{m}, \beta_{m}\right),\left(b_{m+1}, \beta_{m+1}\right), \ldots,\left(b_{k}+r, \beta_{k}\right), \ldots,\left(b_{q}, \beta_{q}\right)
\end{array}\right],
\end{aligned}
$$

when $m+1 \leq k \leq q$.

Identities (??) and (4.5) reduce to a well known generating functions for Fox's H-functions [7, (2.9.10), (2.9.12); p. 25] in the special case when $k=1$ and $k=q$ respectively.

Further if we take $\alpha_{j}=\beta_{k}$ and $\eta=\lambda$ then the result (??) and (4.5) reduce to a well known generating functions for Meijer's G-function [1, (1),(2); p. 213] respectively.

\section{Conclusion}

Besides obtaining the generating relations for the functions in terms of $\mathrm{H}$-functions, the present paper attempts to uncover the group theoretic structure of $\mathrm{H}$-functions. It illustrates how a systematic development can be accorded to the study of $\mathrm{H}$-functions, and then from them particular cases to less general special functions.

\section{References}

[1] A. Erdelyi, Higher Transcendental Functions, Vol. I, McGraw Hill, New York, London, 1953. 
[2] C. Fox, The G and H-functions as symmetrical Fourier kernels, Trans. Amer. Math. Soc. 98 (1961), $395-429$.

[3] B.L.J. Braaksma, Asymptotic expansion and analytic continuous for a class of Barnes integrals, Comp. Math. 15 (1963), 239-341.

[4] W. Miller, Lie theory and special functions, Academic Press, New York, (1968).

[5] W. Miller, Lie theory and Meijer's G-functions, SIAM J. Math. Anal., 5(2) (1974), 309-318.

[6] R. Jain and B.M. Agrawal, Dynamical symmetry algebra of $2 F_{1}$ and Jacobi Polynomials, Journal of Indian Academy of Mathematics, 4(1) (1982), 136-143.

[7] H.M. Srivastava, K.C. Gupta and S.P. Goyal, The H-function of one and two variables with applications, South Asian Pub. Pvt. Ltd., 1982.

[8] R. Jain and B.M. Agrawal, Lie theory and generating functions of some classical polynomials, Vijnana Parishad Anusandhan Patrika, 26(3) (1983), 235-242.

School of Mathematics and Allied Sciences, Jiwaji University, Gwalior - 474011 (M.P.), India.

E-mail: renujain3@rediffmail.com

Department of Applied Mathematics, Madhav Institute of Technology \& Science, Gwalior - 474005 (M.P.), India.

E-mail: jain_dkj@yahoo.co.in

3Department of Mathematics, Rishi Galav College, Morena - 476001 (M.P.), India. 